\title{
Corela
}

Cognition, représentation, langage

13-2 | 2015

Vol. $13, n^{\circ} 2$

\section{Les Ateliers d'Antoine : apports de la Théorie des Opérations Énonciatives en didactique du français à l'école élémentaire}

\section{Lionel AUDION}

\section{(2) OpenEdition}

\section{Journals}

Édition électronique

URL : http://journals.openedition.org/corela/4101

DOI : $10.4000 /$ corela.4101

ISSN : 1638-573X

Éditeur

Cercle linguistique du Centre et de l'Ouest - CerLICO

\section{Référence électronique}

Lionel AUDION, «Les Ateliers d'Antoine : apports de la Théorie des Opérations Énonciatives en didactique du français à l'école élémentaire », Corela [En ligne], 13-2 | 2015, mis en ligne le 25 novembre 2015, consulté le 19 avril 2019. URL : http://journals.openedition.org/corela/4101 ; DOI 10.4000/corela.4101

Ce document a été généré automatiquement le 19 avril 2019

\section{(c) (1) (2)}

Corela - cognition, représentation, langage est mis à disposition selon les termes de la licence Creative Commons Attribution - Pas d'Utilisation Commerciale - Partage dans les Mêmes Conditions 4.0 International. 


\title{
Les Ateliers d'Antoine : apports de la Théorie des Opérations Énonciatives en didactique du français à l'école élémentaire
}

\author{
Lionel AUDION
}

\section{Introduction}

1 Formateur d'enseignants depuis une quinzaine d'années, j'ai souvent fait le constat que la grammaire française scolaire ne répond pas aux besoins des élèves et que de nombreux enseignants du primaire l'enseignent sans lui donner de sens, sans se poser de questions sur son utilité et surtout sans connaissances théoriques précises. Nous savons depuis longtemps que l'essentiel de cet enseignement est une invention scolaire qui ne repose sur aucun fondement théorique (Chervel, 1977), mais l'habitude s'est installée, comme une exception française, et l'on continue pourtant en parallèle à se lamenter sur le fameux «niveau » des élèves, qui ne cesserait de baisser. Cet article s'appuiera sur les conclusions de ma recherche menée auprès d'élèves et de maitres de cycle 3 de 2011 à 2015, lesquelles rejoignent les constats de nombreux didacticiens de la grammaire : une reconfiguration didactique de l'étude de la langue est réclamée depuis plusieurs décennies par un ensemble de chercheurs. Les principales lacunes de cet enseignement sont connues, et je les rappellerai, ainsi que le contexte institutionnel qui n'est pas propice au renouvèlement des pratiques.

2 Tenant compte de ce contexte, je rappellerai l'urgence d'une reconfiguration didactique qui m'a amené à construire un dispositif, testé dans un premier temps en petits groupes, tendant à renforcer la réflexion métalinguistique des élèves de cycle 3 . Après avoir présenté les objectifs de ces ateliers et leur déroulement, ainsi qu'un exemple, je montrerai que cette démarche se fonde nécessairement sur un cadre théorique 
énonciatif. La façon dont Antoine Culioli «travaille la langue » m’a inspiré la manière de problématiser rapidement un fait de langue, et sa théorie m'a permis de mieux appréhender certaines opérations réalisées par les énonciateurs, comme la prise en compte des ajustements dans la coénonciation, ou des opérations métalinguistiques, telles que celles qui sont nécessaires pour déterminer un nom, puisque ce sera l'exemple que je traiterai ici. En effet, il est difficile de montrer à de jeunes enfants ce qu'est une " opération », mais c'est indispensable car c'est, selon moi, la clé d'entrée pour expliquer le fonctionnement du langage. Mon choix s'est porté sur la détermination qui me semble propice à expliquer ce qu'est une "opération", car les enfants ne se posent jamais la question du choix du déterminant, ni même de son rôle. Expliquer que ce choix, qui parait spontané, répond en fait à une logique langagière, est très fructueux en termes didactiques.

Reconceptualiser ainsi la grammaire scolaire dans le premier degré est un défi double, puisque les maitres, polyvalents et sans formation linguistique précise, doivent comprendre et accepter, mais aussi parce que cet enseignement doit rester à la portée de très jeunes élèves. Je présenterai donc, dans un dernier temps, les résultats de cette expérimentation, tant sur les élèves que sur les enseignants qui ont testé mes ateliers métalinguistiques qui sont devenus, en cours d'expérimentation, les «Ateliers d'Antoine » à la demande des maitres-testeurs.

\section{De la nécessité d'une reconfiguration didactique}

Les didacticiens $\mathrm{du}$ français langue maternelle sont convaincus depuis les travaux de Chervel (1977) que la grammaire scolaire, qui n'a pas de fondement scientifique, doit être reconfigurée.

\subsection{La nécessité d'une reconfiguration didactique n'est pas nouvelle}

Dès 1979, un colloque a été organisé à Cerisy par les didacticiens Halté et Petitjean. Il avait pour but de faire le point sur l'entrée de la linguistique à l'école suite au plan «Rouchette » de rénovation de l'enseignement du français, et concluait à la nécessité d'une nouvelle didactique. De l'aveu même de Jean-Claude Chevallier (2011), la linguistique avait été intégrée trop tôt aux programmes scolaires : elle n'était pas encore suffisamment maitrisée par les formateurs d'enseignants ni même, peut-être, par les concepteurs des programmes. Les enseignants les plus anciens se souviennent encore des fameux "arbres de Chomsky" utilisés dès le CE2 pour expliquer aux élèves la construction de la phrase... Si les maitres avaient été suffisamment formés, si l'on avait pu prendre le temps de les convaincre de la nécessité de changer complètement de modèle, cette approche aurait permis de proposer aux élèves une nouvelle description de la langue, plus juste, mais, sans réelle formation ni prise de distance avec les anciens programmes, cette théorie n'a pas remplacé la grammaire scolaire traditionnelle : elle s'y est agglutinée pour accoucher d'un nouveau monstre grammatical. La plupart des enseignants n'a pas compris qu'il s'agissait d'un changement de paradigme et non d'un simple renouvèlement de la terminologie.

6 En 1989, Jean-Paul Bronckart faisait à nouveau des propositions pour une nouvelle didactique du français ; il s'agissait pour lui, citant les propos de Victor Schoeni, 
d'abandonner l'attitude normative qui ignore la langue de l'enfant au nom de la croyance en un français homogène et unique par rapport auquel tout serait déviance (Bronckart, 1989 : 61)

7 L'enquête que j'ai menée ces trois dernières années auprès d'enseignants du premier degré confirme qu'une majorité d'entre eux croit en ce "français homogène et unique » vers lequel il faudrait mener les enfants dont la langue serait forcément "déviante». C'est également une idée ancrée chez de nombreux parents. Lors d'entretiens, beaucoup d'enseignants finissent pourtant par dire eux-mêmes que, finalement, la langue des enfants est « autre » mais pas forcément «moins bien » que le français standard lequel, d'ailleurs, n'existe peut-être qu'à l'école. Simplement, ces enseignants, polyvalents et soumis à de fréquents changements de programmes, ne s'étaient pas posé la question avant que je la leur soumette.

Malgré cet appel au changement, fort et très relayé auprès des formateurs et des enseignants, les pratiques n'ont malheureusement pas changé, au point que Serge Meleuc et Nicole Fauchart (1999) n'hésitaient pas dix ans plus tard à rappeler que la grammaire scolaire, particulièrement la conjugaison, représentait l'un des «noyaux durs de l'immobilisme» dans l'ensemble du paysage didactique. Des propositions didactiques avaient cependant été faites, notamment par Danielle Leeman (1993) qui, elle aussi, constate l'échec de la grammaire traditionnelle et va même jusqu'à penser que les enseignants ont renoncé à enseigner l'étude de la langue (1993: 11). Elle fait donc des propositions aux enseignants qui ne veulent pas

se résigner à venir chaque jour au collège ou au lycée avec pour seule motivation la justification de son (maigre) salaire et horizon l'indifférence amorphe des uns et le chahut des autres. (id: 13)

9 Sa principale proposition, très hardie à l'époque où la "démarche inductive » était proposée aux enseignants comme la seule méthode acceptable, est au contraire de partir de la règle de grammaire, de l'évaluer et, le plus souvent, d'en montrer l'inconsistance. Ces travaux ont eu un peu d'écho dans le second degré, sans pour autant s'imposer, mais n'ont pas été connus à l'école primaire où la démarche était probablement très difficile à mettre en place.

10 C'est en 2002, avec les "nouveaux programmes ", que l'école élémentaire va être touchée puisque, au cycle 3, la matière «français" est remplacée par deux disciplines: la «littérature " et "l'observation réfléchie de la langue française» (O.R.L.F.). Les propositions ministérielles étaient très prometteuses; il s'agissait réellement d'un renversement des pratiques puisque l'axe principal était de partir de la langue des enfants, telle qu'ils la parlaient et l'écrivaient, mais aussi d'autres états de la langue avec lesquels les professeurs étaient invités à construire des corpus. Ces "observables" donnaient matière à la construction de règles de fonctionnement. Quelques didacticiens ont accompagné cette réforme, mais assez tardivement, notamment Michelle Ros-Dupont (2006) qui explique aux maitres comment transformer leurs élèves en "observateurs" (par exemple à propos des variations en temps et en personne des verbes, qui permettent de les repérer).

11 Encore un peu plus tard, l'équipe de Ghislaine Haas (2007), en Bourgogne, propose d'adapter à l'enseignement de la grammaire en premier degré les théories de Britt MariBarth sur l'abstraction, en utilisant les « exemples oui » et les « exemples non ». L'équipe de Haas avait également proposé de nouvelles approches de l'orthographe (2004), notamment autour des ateliers de négociation graphique, idée proposée à la même 
époque par Danièle Cogis (2005) qui aide également les enseignants en leur proposant de nouveaux outils, comme « la phrase dictée du jour ».

Les propositions existent donc, la formation initiale les relaie abondamment, mais les pratiques dans les classes évoluent peu, sauf en orthographe ou la négociation graphique gagne peu à peu du terrain. En 2008, un ministre décide que la parenthèse de « l'O.R.L.F. » doit se refermer et invite les enseignants à revenir à des pratiques plus traditionnelles, que la plupart d'entre eux n'avait de toute façon pas abandonnées.

Pendant toute cette période, le didacticien Claude Vargas qui observait cette absence de changement de pratiques malgré les programmes de 2002, n'a pas cessé d'appeler, lui aussi, à une transformation de l'enseignement de la grammaire. En 2009 encore, il dénonçait

les incertitudes, les contradictions, les incohérences, voire les aberrations issues de la recomposition didactique des années 70-80 [et appelait à] une reconfiguration didactique des savoirs grammaticaux, qui permettrait de faire de la grammaire scolaire un objet cohérent. (Vargas, 2009, p32)

La recherche que je mène depuis 2012 m'a permis d'observer ces pratiques figées (le verbe est le mot de la phrase qui désigne l'action ; le C.O.D. répond à la question « quoi ? », etc.) et, au travers d'entretiens, ces représentations fausses de la langue et de son étude ( «la langue, c'est l'écrit, de toute façon. L'oral, c'est pareil avec quelques petits mots et des interjections en plus»; "la grammaire, c'est la grammaire, il n'y a pas cinquante théories, ça n'a jamais changé »).

Le tableau que je brosse des pratiques de l'enseignement de la grammaire dans le premier degré est pessimiste : peu de connaissances sur la langue par les enseignants, du fait de la polyvalence et d'une formation trop brève, méthodes très rigides et très " descendantes " malgré des tentatives inductives, mais souvent trop guidées, connaissances des élèves parfois suffisantes mais pas assez « efficaces » lorsqu'ils produisent de l'écrit. Ce constat est largement partagé par les didacticiens actuels: outre Bronckart, cité plus haut, Patrice Gourdet a également observé « le décalage entre les objectifs d'apprentissage et les connaissances des enseignants titulaires » (2009: 301) ; Caroline Masseron dresse également un bilan accablant et conclut :

C'est devant de tels constats que nous sommes conduite à parler d'urgence dans le travail de réflexion collective à mener si l'on veut sortir l'enseignement de la langue de son ornière (2001: 213)

Tout récemment, Marceline Laparra titrait sa conférence « Une discipline en crise... ou une discipline en ruines?» (avril 2015). En tant qu'auteure de propositions didactiques depuis longtemps, notamment au sein de la revue Pratiques, elle pense, dans une véritable remise en question du travail réalisé depuis de longues années, et en associant ses collègues, qu'ils ont "produit de l'inaudible ou de l'audible qui a été déformé » et s'interroge sur ce que les didacticiens doivent produire comme discours commun à l'adresse des enseignants pour les « re-stabiliser ».

Voyons à présent plus en détail les principales lacunes de l'enseignement de la grammaire à l'école élémentaire.

\subsection{Principales lacunes du modèle actuel}

18 a) Le défaut le plus grave est sans doute l'absence de réflexion sur la nécessité d'enseigner la grammaire d'une langue maternelle. Cette question est complètement absente des 
débats, et pourtant notre grammaire scolaire est une exception française. La proposition que je ferai ci-dessous tente de montrer qu'une réflexion métalinguistique est indispensable aux enfants pour étayer l'apprentissage de leur langue et sans doute aussi pour essayer d'égaliser les chances des élèves confiés à l'école. Mais en l'absence de cette réflexion, il faut nous contenter d'une finalité orthographique, qui semble être l'attente de la société. Si c'est vraiment le cas, une grande partie du dispositif grammatical proposé dans les programmes de l'école élémentaire est inutile.

Les autres lacunes de notre modèle sont connues et largement partagées, je les rappellerai rapidement :

b) La grammaire scolaire est très classificatoire, ce qui ne serait pas très grave si l'on mettait du sens derrière toute la terminologie utilisée, mais les pratiques observées montrent que de nombreux professeurs du premier degré apprennent à leurs élèves à « coller des étiquettes » (parfois au sens propre) de classe ou de fonction, sans inviter à la réflexion. On connait les conséquences de ces classifications mécaniques, mais on n'insiste peut-être pas suffisamment sur le fait que l'une d'entre elles est la mauvaise préparation des élèves à l'apprentissage d'une langue étrangère. Or, les enfants commencent cet apprentissage dorénavant très tôt, parallèlement à l'enseignement de la grammaire du français : si l'un et l'autre étaient complémentaires, au lieu d'être ressentis comme complètement différents, ce serait une grande avancée.

21 c) La grammaire scolaire est circonscrite à la phrase écrite, et cela dans le cadre d'une langue presque spécifique, "découpée " dans la langue française: une langue que, finalement, on ne rencontrerait que rarement à l'extérieur de l'école, ce qu'Antoine Culioli appelle « un champ opératoire aseptisé » (Bouquet, 2004, p138).

d) Les savoirs grammaticaux que les élèves se sont construit seuls, progressivement, ceux que Fabienne Calame-Gippet (2008) appelle les "connaissances ignorées", ne sont presque jamais pris en compte par l'école. Pourtant, lorsqu'un enfant de maternelle raconte qu' " un loup *coura après les petits cochons ", un énorme travail grammatical a déjà été réalisé (l'enfant a par exemple compris comment se construit ce temps par rapport à un modèle fréquent et à quel moment du récit il faut l'utiliser).

e) La grammaire scolaire fait semblant, depuis les années 80 , de reposer sur une démarche inductive et même parfois sur une démarche de problématisation. Mais les séances d'étude de la langue les plus souvent pratiquées ne pointent aucun problème de langue, font observer aux élèves des phrases " authentiques " prélevées presque au hasard ici ou là, au lieu de construire un énoncé que les élèves pourraient faire proliférer, afin de bâtir un problème.

24 Le didacticien du français est donc placé devant la nécessité d'offrir aux praticiens de nouveaux outils qui permettraient de combler ces lacunes.

\section{Ma proposition : des ateliers métalinguistiques}

J'ai conçu un dispositif à l'adresse des maitres de Cycle $3^{1}$ proposant un outil simple pour les élèves, leur permettant de réfléchir à des faits de langue problématisés, et une fiche de savoirs pour eux-mêmes. 


\subsection{Des ateliers déconnectés des séances de grammaire}

La démarche que je propose s'adresse à des groupes à faible effectif, animés par le maitre, ce qui rend difficile sa mise en place en classe entière, même dans un dispositif de groupes autonomes, que j'ai testé. C'est pourquoi je la propose aux maitres pendant les temps d'Activités Pédagogiques Complémentaires (A.P.C.) ${ }^{2}$. En groupes de 6 à 8 , les élèves sont invités à débattre sur un problème (par exemple : « des » est-il toujours le pluriel de « un, une » comme le prétend la règle scolaire? Voir exemple développé plus bas). Le maitre anime les premiers ateliers puis est rapidement invité à déléguer cette animation à un élève "chef de projet ", qui a également la responsabilité de noter les résultats du travail sur un carnet de recherches, en prenant les notes dictées par ses camarades.

Cette approche nécessite une mise en place sur un temps plus long et ne peut donc pas couvrir l'intégralité du programme. Elle demande un travail d'adaptation important aux maitres, c'est aussi pourquoi il ne serait pas possible de la proposer en substitution des séances de grammaire traditionnelle : je m'inscris ici dans les pas de Goigoux et Cèbe (2011) qui ont théorisé l'élaboration d'artéfacts didactiques et qui insistent sur la différence entre le « souhaitable » et le « raisonnable ».

Bien évidemment, le contenu de l'atelier travaillé tel jour sera choisi en fonction de la progression-programmation des séances de grammaire, mais il peut être intéressant, parfois, de choisir un atelier en rupture avec ce qui est vu en parallèle pendant les cours d'étude la langue; je pense notamment à des ateliers prévus pour faire réfléchir les élèves sur les ressemblances et les différences entre le français et l'anglais (par exemple : avoir est-il un verbe d'état?). Comme on le voit, ces ateliers peuvent être perçus et utilisés comme des moyens de remédiation, mais, à la façon d'un cheval de Troie, ils ont surtout pour objectif de convaincre les maitres de la nécessité des pratiques métalinguistiques et de les amener à adopter la méthode pour s'en inspirer également dans les séances en classe entière : dans le cadre de la " genèse instrumentale » étudiée par Rabardel (1995), il s'agit de faire en sorte que "l'instrument » devienne "outil»; j'ai donc étudié cette genèse et me suis aperçu que certains maitres-testeurs détournaient les ateliers pour les proposer, modifiés, en classe entière : l'instrument que j'avais proposé était devenu un outil, de la même façon que certains utilisateurs du couteau s'en servent comme d'un tournevis, ce qui a donné des idées aux fabricants de couteaux.

\subsection{Les Ateliers d'Antoine}

Afin de motiver les jeunes enfants, il est utile de les placer face à un problème, comme ils y sont déjà habitués en mathématiques, en sciences, parfois en histoire et géographie. La durée des ateliers étant très limitée, cette problématisation doit être très rapide, c'est pourquoi je me suis inspiré d'une démarche d'Antoine Culioli qui permet cette rapide mise en situation problème :

Ce qui a été ajouté, c'est la recherche dans ces manipulations de ce qui est impossible et après, on va chercher ce qui bloque, ce qui empêche une phrase d'être acceptable ou non et quelles sont les manipulations supplémentaires qui vont faire que ça va redevenir acceptable [...]. Vous fabriquez un carré d'énoncés de sorte que vous ayez trois possibles et un impossible: Le chien est un mammifere; Les chiens sont des mammiferes; Un chien est un mammifere ; *Des chiens sont des mammiferes. [...]. Il faut travailler à intonation constante. C'est ainsi qu'on construit des données. (1984:5). 
30 programme de grammaire du Cycle 3 ; je les ai testés et fait tester puis les ai améliorés en tenant compte des remarques; c'est ainsi par exemple que mes carrés sont devenus « carrés-problèmes ", ce qui semble plus facile et plus habituel pour les élèves.

Le moment important, en classe, n'est pas celui où les enfants, individuellement, remplissent le carré (deux énoncés proches, une consigne de transformation, et deux cases vides pour écrire les réponses) mais le débat d'acceptabilité qui suit cette prolifération d'énoncés et valide ceux qui ont frayé, débat qui se termine par la proposition d'une "règle", consignée sur le carnet de chercheur. Selon les cas, la règle ressemble à celle que l'on trouve dans les manuels mais, le plus souvent, elle la modifie ou la précise (c'est le cas pour le pluriel de «un, une»). La règle à laquelle les enfants arrivent n'est pas importante en soi : l'objectif des ateliers est bien le débat lui-même, le processus métalinguistique qui fait bouger les représentations. Il faut bien, toutefois, arriver à une trace écrite de la conclusion de ce débat (les enseignants que j'ai interrogés ou ceux qui ont testé les ateliers sont très fermes sur cette exigence). Nous savons que l'approche de la grammaire par règles n'est pas fructueuse, c'est pourquoi nous la désacralisons en permettant aux élèves de les construire eux-mêmes afin, à moyen terme, de pouvoir s'en affranchir peut-être complètement, mais le cadre institutionnel, les textes officiels et les programmes sont pour le moment très contraignants à cet égard.

Ce dont les maitres s'aperçoivent pendant ces ateliers, et c'est très important, c'est le rôle de la contextualisation des énoncés (quel contexte peut-on trouver pour que cet énoncé soit acceptable ?) qui permet leur interprétation, laquelle montre aux enfants l'étendue de leur pouvoir sur le langage. Faire proliférer un énoncé, c'est prendre conscience de l'étendue des possibles qu'offre la langue, mais aussi de l'importance de la contextualisation, ce que la grammaire de phrase traditionnelle ne permet pas.

Afin d'aider les maitres à animer les ateliers et guider le débat d'acceptabilité, mais aussi pour les équiper en connaissances théoriques, chaque fiche est accompagnée d'une page de «savoirs du maitre " résumant ce que les enseignants doivent savoir et indiquant précisément à quelle théorie ces savoirs font référence, car il est devenu incontournable de «re-conceptualiser » la grammaire scolaire qui a l'habitude de ne pas dire d'où elle vient (Bronckart, 2015). Plutôt que de proposer une formation (initiale ou continue) sur les théories de l'énonciation, j'ai donc testé la proposition de Goigoux et Cèbe sur les « savoirs pour l'action »:

Nous pensons (...) plus efficace d'agir directement sur les pratiques des enseignants pour modifier leurs conceptions, plutôt que l'inverse (2011)

C'est ainsi que ces auteurs ont proposé par exemple le manuel «Catego » (Hatier, 2004) pour les classes de maternelle, qui remporte un très grand succès et a modifié les pratiques. Mes fiches "savoirs du maitre » ont montré auprès de professeurs des écoles en formation à l'E.S.P.É. de Nantes qu'elles atteignaient leur but, alors que des séances de cours traditionnelles, les années passées, n'avaient pas modifié les pratiques des professeurs stagiaires.

\subsection{Le « carré-problème » et le débat d'acceptabilité}

Les Ateliers d'Antoine sont proposés pour les élèves de CM1 et CM2, dans le cadre du dispositif d'aide que sont les Activités Pédagogiques Complémentaires (APC) proposées

Corela, 13-2 | 2015 
une fois par semaine à des élèves choisis par l'enseignant. Ces Activités sont réalisées le plus souvent sur le temps du midi et durent une vingtaine de minutes. Il faut donc que l'activité proposée problématise très rapidement un fait de langue afin de permettre aux élèves de pouvoir y réfléchir et tenter de le résoudre. C'est pourquoi en m'inspirant d'Antoine Culioli, j'ai proposé la technique du « carré problème » : dans les cases du haut figurent deux énoncés assez proches ; une consigne propose une transformation : l'un des énoncés se transforme facilement alors que l'autre pose problème. C'est à partir de ce problème que le maitre lance le débat d'acceptabilité. Voici un exemple de carré, à partir de la notion de détermination que nous avons abordée plus haut :

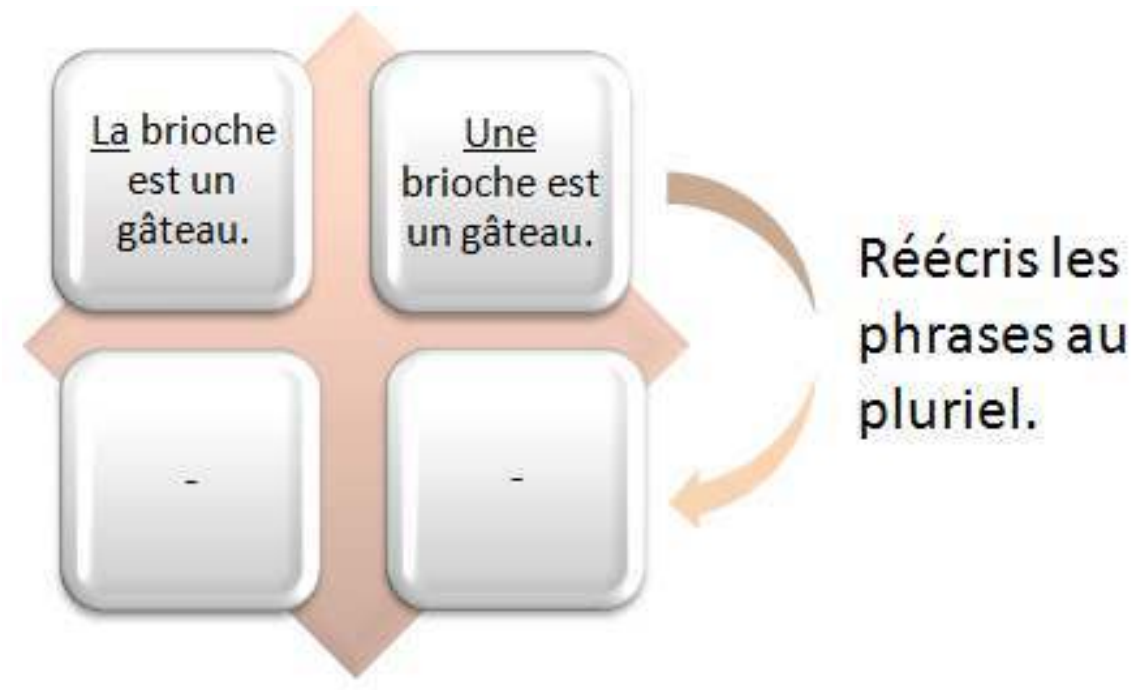

Le premier énoncé est facilement transposé au pluriel : « Les brioches sont des gâteaux ». Pour le second énoncé, les élèves les plus « scolaires », ceux qui connaissent et appliquent les règles, savent que «le pluriel de une est des » et proposent : «Des brioches sont des gâteaux" (énoncé 2a); les autres écrivent spontanément "Les brioches sont des gâteaux » (énoncé $2 b$ ). S'ensuit alors un débat d'acceptabilité : les deux énoncés sont grammaticalement corrects, mais les deux sont-ils acceptables?

Lorsque je propose ce carré, les réactions des élèves sont de deux ordres : d'abord la recherche d'un contexte d'acceptabilité pour 2a, puis un « conflit de loyauté » lorsqu'ils finissent par préférer $2 b$. Voici un extrait de débat mené dans une classe de CM1, illustrant la recherche d'un contexte d'acceptabilité :

FLORIAN : ça fait bizarre, quand même; c'est comme si y'en avait d'autres qui seraient pas des gâteaux.

JÉRÉMY : Ah bah oui, je sais, y’a des brioches qui sont pas des gâteaux : quand on a un gros ventre, comme mon papy, on dit qu'il a de la brioche.

FLORIAN : alors, on peut dire : des brioches sont des gâteaux, mais d'autres sont des ventres.

LOÉVA : n'importe quoi ! personne dirait ça...

FLORIAN : peut-être, mais ça peut se dire !

On note dans la réplique de Jérémy la volonté de trouver un contexte d'acceptabilité pour l'énoncé « correct » mais non acceptable dans le sens habituel de «brioche ». Le principe étant que l'enseignant intervienne le moins possible, il a cependant pu noter, afin d'y 
revenir plus tard, de rappeler à ses élèves l'importance de la contextualisation et la prise en compte nécessaire des contraintes sémantiques autant que syntaxiques, ce qui n'est pas habituel dans le discours grammatical ordinaire de l'école.

Les deux dernières répliques sont également très intéressantes : "personne dirait ça " montre que 2a n'est pas acceptable. En effet, "des brioches sont des gâteaux » sousentend que d'autres brioches seraient autre chose. "Peut-être, mais ça peut se dire " montre que le français offre cette potentialité, elle est correcte; c'est un bon exemple pour faire réfléchir les élèves sur le fait que la grammaire ne dit rien de ce qui est acceptable, le "trésor» qu'évoquait Saussure et que chacun de nous se construit progressivement.

La suite du débat montre que les enfants ont le sentiment de violer une règle que leur enseignante (Pauline) leur a apprise :

$\mathrm{C}$ : bon, revenons à notre question : est-ce que « les brioches sont des gâteaux » est

le pluriel de «Une brioche est un gâteau »?

JEANNE : ben ouais, autrement ça serait « des brioches sont des gâteaux » et, à part si c'est le ventre, c'est pas français.

AWENA : oui, mais normalement « les » c'est pas le pluriel de " une »; c'est « des », on l'a vu avec Pauline.

MAXENCE : ah oui, ça c'est vrai, même qu'on l'a marqué sur le cahier.

$\mathrm{C}$ : alors, nous voilà face à un problème... comment le résoudre ?

MAXENCE : ben faut changer la règle, mais faudra le dire à Pauline.

$\mathrm{C}$ : et que proposerais-tu, comme règle?

MAXENCE :...

FLORIAN : « des » est le pluriel de " un » et « une » sauf exceptions, et on note les exceptions où c'est « les ».

JEANNE : et tu les connais, toi, toutes les exceptions?

FLORIAN : ben non, pas toutes... Y'en a beaucoup ?

$C$ : je pense que la liste serait trop longue. Est-ce que l'on pourrait trouver une autre façon de formuler cette règle ? Allez, cherchez un peu, au brouillon.

LOÉVA : (au bout de deux minutes environ) : «des » est le pluriel de " un » et « une » sauf si le sens de la phrase veut qu'on mette « les ».

C : êtes-vous d'accord, les autres ? Y a-t-il d'autres propositions?

41 En un vingtaine de minutes, ces jeunes élèves ont été confrontés à un problème et l'ont résolu en imaginant une règle de grammaire différente de celle qu'ils ont apprise, mais rendant mieux compte de la réalité de la langue. Rappelons que si la formulation de la règle peut apparaitre aux élèves comme le but des Ateliers d'Antoine, nous savons que c'est le débat qui précède qui est important; dans la règle proposée ici, je retiens que les élèves ajoutent un critère sémantique à l'habituel critère syntaxique : pour des apprentis chercheurs de 9 et 10 ans, un grand pas a été franchi. 
La réflexion métalinguistique des élèves est toujours plus riche que ce qu'imaginent les enseignants : ce fut le cas de l'enseignante de cette classe qui fut très surprise de la qualité de réflexion de ces élèves qui fréquentaient l'atelier parce qu'ils étaient «faibles » en français.

\section{Les Ateliers d'Antoine sont ancrés dans le cadre théorique de l'énonciation}

\subsection{Importance des ajustements}

43 Comme on l'a vu, c'est une réflexion d'Antoine Culioli sur la conception des "carrés d'énoncés » qui m'a permis de trouver le dispositif que je cherchais. Ce n'est évidemment pas la seule raison pour laquelle je l'ai appelé «Ateliers d'Antoine » : il fallait bien sûr trouver un nom adapté au public («ateliers métalinguistiques» aurait été immédiatement rejeté par les maitres) et, surtout, la réflexion sur la langue qui sous-tend cet outil se nourrit de la Théorie des Opérations Énonciatives. Il n'est pas question pour moi d'entreprendre une transposition didactique de l'ensemble de cette théorie à destination de l'enseignement de la langue maternelle dans le premier degré, ce qui serait trop ambitieux et sans doute peu pertinent, mais de prendre en compte certains éléments, comme le domaine notionnel pour les Ateliers portant sur le vocabulaire, ou la nécessité de rappeler que l'étude de la langue ne peut se faire qu'en situation, en tenant compte du contexte, comme on l'a vu, mais aussi du destinataire et des ajustements nécessaires.

Les enseignants ont des connaissances à ce sujet, issues du lycée, se résumant souvent au "schéma de Jakobson », rarement contesté car impensé dans l'enseignement du français, mais ne rendant pas du tout compte de la complexité des échanges, complexité qu'Antoine Culioli a tenté de résoudre, en affirmant d'abord que, contrairement à ce qui est le plus souvent avancé, un «destinateur» n'envoie pas un message à un « destinataire » qui, facilement, le décoderait avec son transcodeur :

Nous avons alors une métaphore qui est, en gros, celle du commutateur : je suis, à un moment donné, émetteur, j'envoie un message, et il y a un récepteur en face ; le récepteur, muni du code commun, décode; puis il tourne dans l'autre sens le bouton et devient lui-même émetteur (...). Cela ne marche pas du tout ainsi. (Culioli 1990 : volume 1. p25).

La réalité est que le message se co-construit entre les coénonciateurs A et B en s'ajustant à ce que $A$ sait ou croit savoir de $B$ : $A$ se réfère d'abord à un coénonciateur interne (noté «CoÉ Int A » dans le schéma ci-dessous), sorte de double ou d'image fantasmée de B : ce que $A$ sait ou croit savoir de $B$; dans ce premier échange, $A$ procède déjà à des

ajustements (l'ajustement est représenté par le symbole

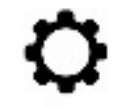

dans la figure cidessous). A s'ajuste également à la langue, afin de choisir, dans l'infini des possibilités, les mots et leurs arrangements afin de bien dire ce qu'il veut dire. Le message est envoyé au coénonciateur B (noté « CoÉ Ext de A ») qui se livre à des ajustements lors de la réception.

Le chemin est identique mais inverse pour la réponse. On peut donc figurer ainsi un échange entre A et B, et s'apercevoir qu'on est bien loin du schéma de Jakobson ${ }^{3}$ : 


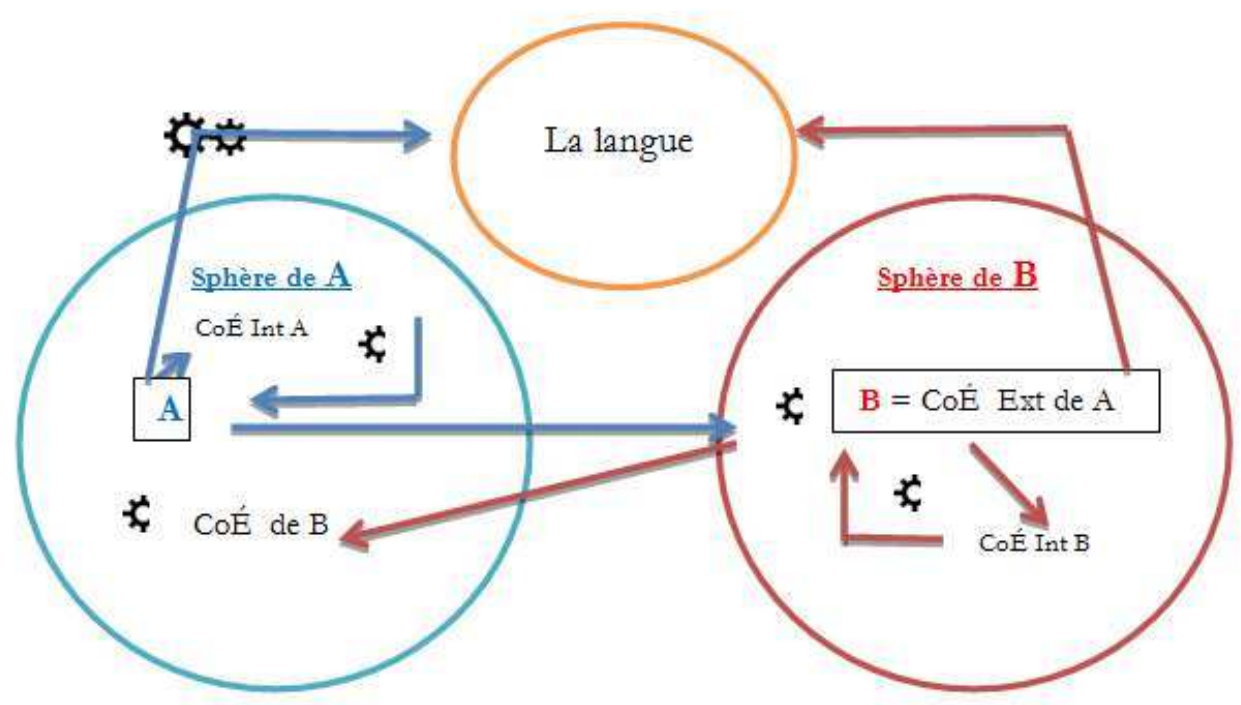

Lors des débats d'interprétations auxquels j'ai assisté ou que j'ai animés, les élèves ont à plusieurs reprises eu l'occasion de s'apercevoir de la fréquence des ajustements, par exemple en reformulant leurs propositions («mais non, tu comprends pas, je recommence »), ou en s'apercevant que « brioche » réfère a plusieurs objets (le gâteau et le ventre). Dans un atelier de vocabulaire consacré au zonage, les élèves ont débattu pour savoir si, par exemple, « danse » était à l'intérieur du " domaine du sport », à la frontière ou à l'extérieur, en plaçant des mots étiquettes sur un carton ovale symbolisant le domaine :

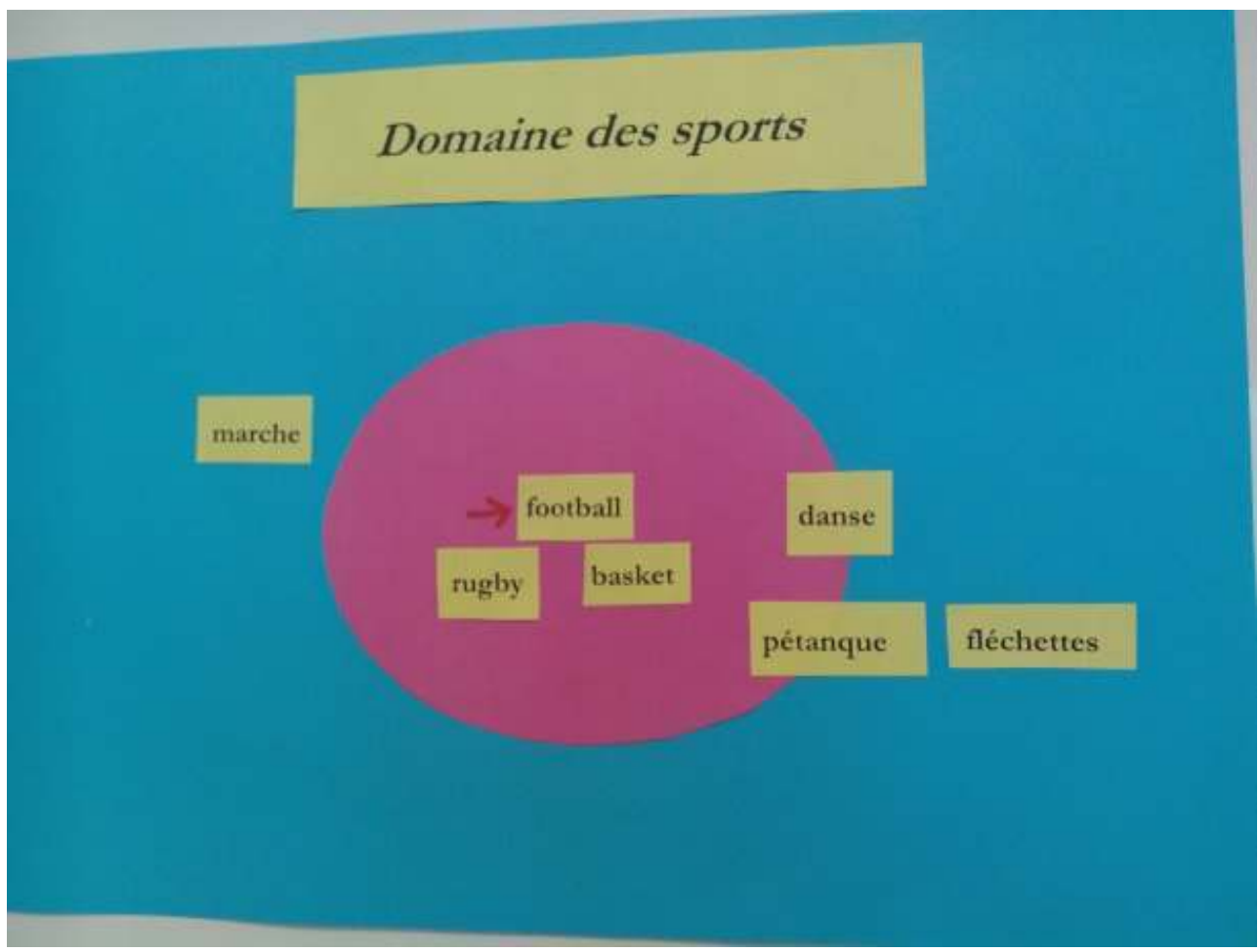

47 Ils se sont ainsi rendu compte que, d'une part, le même mot ne renvoyait pas au même domaine pour chacun d'entre eux mais que pourtant, d'autre part, chacun employait le même mot en l'ajustant. 


\subsection{Nécessité de reconceptualiser les savoirs des enseignants}

Les enseignants n'ayant pas tous la formation nécessaire pour animer ces ateliers, ou pour tirer les enseignements nécessaires des propos échangés par les élèves lors des débats, la fiche « savoirs du maitre ", accompagnant les Ateliers, est indispensable.

L'Atelier « brioches » présenté plus haut est ainsi l'occasion de proposer aux maitres une réflexion sur les articles génériques et spécifiques, mais aussi et d'abord sur l'opération de détermination : comment construit-on le groupe déterminant + nom ? Pourquoi le nom a-t-il besoin d'être déterminé ?

$\mathrm{Au}$ niveau de l'enseignement primaire, on peut expliquer l'extraction (sans employer le terme lui-même) par la nécessité de créer dans la langue une occurrence de notion: Expliquons aux enfants que le nom "rose » n'existe pas... Surprise des élèves qui sont bien convaincus du contraire! Pourtant, en langue, on trouve " une rose ", "la rose ", « ma rose », etc., mais très rarement « rose » et seulement dans des contextes très précis qu'il est facile d'expliquer (légende de photographie, titre, vocatif, etc.). Cette notion-là ne se trouve que dans le dictionnaire ; pour lui « donner vie » il faut extraire une occurrence, au moyen de un ou une. Les mots prennent vie en s'envolant du dictionnaire.

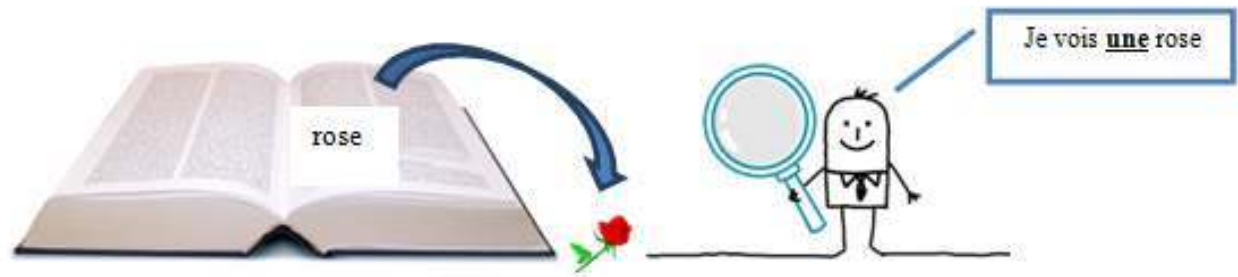

51 Lorsque je veux utiliser un nom qui est "déjà là ", car il a déjà été créé dans la coénonciation (opération de «fléchage»), j'utilise l'article défini ou un autre déterminant. Contrairement à ce que dit le plus souvent le discours scolaire, l'occurrence « déjà là » n'a pas forcément été rencontrée "avant » dans le texte, mais elle peut être connue des coénonciateurs: je peux par exemple dire «ce soir, je vais chercher les enfants à l'école » : mon coénonciateur sait de quelle école il s'agit, celle qui existe " déjà » dans l'espace de la coénonciation. Certains manuels donnent des exemples proches (« le directeur de l'école », « le Président de la République ») mais en n'expliquant jamais la raison de l'emploi du défini.

2 Enfin, je peux parcourir l'ensemble des occurrences sans m'arrêter sur une en particulier; en français, on utilise dans ce cas-là l'article défini (« la rose est une belle fleur ») ou indéfini (« une rose sent toujours bon »).

Je m’aperçois donc que je peux rencontrer deux «un » différents: le générique et le spécifique. bien que la grammaire scolaire affirme que le pluriel de un est des, ces deux un ont-ils le même pluriel ? Voilà un exemple de problème posé au didacticien. Depuis une vingtaine d'années que j'assiste à des séances de français à l'école élémentaire, je n'ai pas souvent eu l'occasion de voir des maitres aborder cette question. Ce serait pourtant l'occasion, là encore, d'une approche par l'interprétation : le « un » générique en position sujet s'interprète-t-il de la même façon que ses concurrents? Pourquoi choisir de dire « une baleine est un mammifère » plutôt que « la baleine... » ou « les baleines... »? Voilà des questions que les élèves affrontent spontanément lors des débats d'acceptabilité et ce 
sont toujours des moments importants de prises de conscience sur l'étendue des possibilités qu'offre la langue.

\section{Des résultats encourageants} réfléchir sur la langue, sur les infinies possibilités qu'elle offre, qu'il faut donc connaitre et savoir utiliser pour dire ce que l'on veut dire, et pas autre chose qui serait mal interprété.

- L'outil créé devait me servir en formation et être assez motivant pour que les professeurs des écoles stagiaires s'en emparent (cf les « savoirs pour l'action»). animés moi-même que dans ceux dirigés par les maitres-testeurs. Le principal enseignement est que les élèves en difficulté se laissent enrôlés plus facilement dans les activités, par rapport aux méthodes traditionnelles; c'est ce que me dit la première enseignante ayant accepté de tester les élèves («MT1») :

$\underline{\mathrm{C}}$ : et ces élèves en difficulté, vous m'avez écrit dans un bilan d'atelier qu'ils participaient bien et vous avez même dit pour l'atelier 12 : «ils ont compris (même les plus faibles !) les différents rôles que jouaient les auxiliaires ».

MT1 : oui c'est ça. J'ai trouvé ça hallucinant quoi parce que en classe sur un exercice là-dessus ils auraient même pas essayé et là ben ça a marché ils se sont pris au jeu comme s'ils avaient oublié qu'ils faisaient de la grammaire.

L'autre point positif de ce bilan est que les élèves ont vraiment eu conscience de construire leurs savoirs en se construisant une représentation de la langue : ce n'est pas l'école qui leur impose un modèle. Je retrouve cette idée dans divers entretiens :

comment dire, vous voyez quand on fait de la grammaire en classe, ils savent bien qu'on va arriver à quelque chose qui était déjà préconstruit, qui est déjà écrit dans un livre, tout ça. Là, ils m'ont donné l'impression, enfin pas tous, hein, ils m'ont donné l'impression, euh, que voilà, c'est ça, qu'ils pouvaient arriver à quelque chose qui n'était pas écrit d'avance et que ça allait quand même être pris en compte. Voilà c'est ça je crois. Moi j'trouve ça génial, j'ai pas eu cette impression-là autrement, sauf en sciences des fois. (MT1-9)

MT2, professeur-stagiaire également, partage cette idée :

on part des représentations des élèves mais pas pour la frime et les amener de gré ou de force là où on veut qu'ils aillent. Vous voyez, c'est ça qui m'agace dans la pédagogie qui fait croire que tout vient des élèves mais tout est déjà écrit sur le manuel et si t'arrives pas à ce qui était prévu, t'es nul. (MT2-12).

Sur l'année 2014-2015, trois professeurs-stagiaires ont accepté de devenir «maitrestesteurs » avec leurs élèves. Ils disposaient de 13 «Ateliers d'Antoine » complets (fiches élèves et « savoirs du maitre » rappelant les notions théoriques nécessaires pour proposer l'atelier aux élèves) mis à leur disposition sur un site internet spécifique ${ }^{4}$, uniquement destiné à télécharger les fiches au fur et à mesure que je les proposais ou modifiais, et à faire remonter leurs impressions. En cours d'année, des entretiens collectifs et individuels ont également permis d'évaluer l'appropriation de l'artéfact.

61 Du côté des professeurs en formation, donc, les premiers résultats obtenus sont très encourageants : forte adhésion des professeurs stagiaires et demandes d'informations de 
la part de leurs tuteurs ; de plus, on m'a demandé de proposer une formation continue sur ces ateliers. Les stagiaires impliqués dans la recherche ont été beaucoup plus actifs et intéressés durant les cours de français donnés à l'institut de formation, manifestant euxmêmes une attitude métalinguistique que je n'avais pas rencontrée jusqu'à présent dans ma carrière de formateur.

\section{Conclusion}

Mon objectif n'est pas de bouleverser les pratiques des enseignants en classe: pour reprendre les propos de Roland Goigoux et Sylvie Cèbe, même si c'est «souhaitable », ce ne serait pas « raisonnable » car trop éloigné de leurs habitudes de travail ; le but est de proposer à tous les élèves des temps métalinguistiques et aux élèves repérés en difficulté une autre approche de la grammaire, en leur permettant de construire les règles euxmêmes (et de comprendre ainsi leur nécessité et l'infini des possibilités qu'offre la langue).

63 L'objectif ultime reste bien, à l'école élémentaire, la maitrise du français écrit correct, puisque c'est l'attente principale de l'école, comme le rappellent les travaux de Reuter sur Goody: «l'école et l'écriture sont inextricablement liées depuis leurs origines (d'où la difficulté de le séparer à des fins d'analyse) » (cité par Reuter, 2006 : 133).

L'écriture, telle qu'elle est modélisée par Goody, «avec l'accent porté sur la distance, l'abstraction, l'analyse, la réflexivité » (idem) est donc le médium privilégié pour construire les savoirs. À l'école élémentaire, l'écriture n'est pas encore suffisamment maitrisée, d'où la nécessité de passer par les ateliers métalinguistiques qui permettent une réflexion sur la langue, une prise de distance, qui facilitent le passage de l'oral à l'écrit, car je fais le pari avec Antoine Culioli que le travail au niveau métalinguistique stimule l'activité cognitive. Le "carnet de chercheur » qui est intégré au dispositif des Ateliers d'Antoine a cet objectif.

\section{BIBLIOGRAPHIE}

BOUQUET, Simon (2004) Antoine Culioli, un linguiste devant les textes saussuriens. Les cahiers de l'Herne $\mathrm{n}^{\circ} 76$. Paris.

BRONCKART, Jean-Paul, (1989), « Du statut des didactiques des matières scolaires », in Langue française $\mathrm{n}^{\circ} 82$, Paris : Larousse (pp 53-66)

BRONCKART, Jean-Paul, (2015), « Que faire de la grammaire et comment la faire », colloque « les 40 ans de Pratiques », Metz, 10-04-2015.

CALAME-GIPPET, Fabienne (2008), « Entrer dans l'analyse grammaticale de la langue à l'école : les ressources ignorées des élèves ", in Penloup, Marie-Claude (dir), Les connaissances ignorées;

approche pluridisciplinaire de ce que savent les élèves. Paris : INRP (pp 7-104). 
CHERVEL, André (1977), Et il fallut apprendre à écrire à tous les petits Français. Histoire de la grammaire scolaire. Pars : Payot.

CHEVALLIER, Jean-Claude : Communication au colloque annuel de la SHESL, Paris, janvier 2011. COGIS, Danièle (2005) Pour enseigner et apprendre l'orthographe (école, collège), Paris : Delagrave. CULIOLI, Antoine (1984) Théorie du langage et théorie des langues (G. Serbat, éd.). Actes du colloque E. Benveniste aujourd'hui, pp 77-87

CULIOLI, Antoine, (1990) Pour une linguistique de l'énonciation, Tome 1. Gap : Ophrys

CULIOLI, Antoine \& NORMAND, Claudine (2005), Onze rencontres sur le langage et les langues. Gap : Ohrys.

GOIGOUX, Roland \& CEBE, Sylvie (2011), « Un autre rapport entre recherche, pratique et formation. Les instruments didactiques comme vecteur de transformation des pratiques des enseignants confrontés aux difficultés d'apprentissage des élèves ». in Talbot, L. (Ed.) : Les pratiques d'enseignement et la prise en compte de l'hétérogénéité des élèves. Bruxelles : De Boeck. GOURDET, Patrice (2009) L'enseignement de la grammaire à l'école élémentaire : le cas du verbe en CE2, thèse de doctorat, Paris et Rome.

HALTÉ Jean-François \& PETITJEAN, André (dir), (1979) Pour un nouvel enseignement du français. Actes du colloque de Cerisy. Metz : Pratiques, numéro spécial.

HAAS, Ghislaine et al. (2004) L'orthographe au quotidien, Paris, Dijon : SCEREN-CRDP de Bourgogne. HAAS, Ghislaine et al. (2007) Classes et fonctions grammaticales au quotidien, Paris, Dijon : SCERENCRDP de Bourgogne.

LAPARRA, Marceline (2015), " Une discipline en crise... ou une discipline en ruines? », communication au Colloque Quarante ans de Pratiques, Metz, 08 avril 2015.

LEEMAN, Danielle (1993), La grammaire ou la galère, Paris : Bertrand-Lacoste.

MASSERON, Caroline (2001) Note critique sur les exercices de grammaire. Pratiques 111/112. (pp 209-236).

MELEUC, Serge \& FAUCHART, Nicole, (1999) Didactique de la conjugaison. Paris : Bertrand-Lacoste. RABARDEL, Pierre (1995) Les hommes et les technologies, une approche cognitive des instruments contemporains. Armand Colin, Paris

REUTER, Yves (2006) A propos des usages de Goody en didactique, Pratiques 131/132 (pp 131-154). ROS-DUPONT, Michelle (2006) Observation réfléchie de la langue, Cycle 3 Paris : Nathan.

VARGAS, Claude (1992) La grammaire pour enseigner, Paris : Armand-Colin

VARGAS, Claude (2009) « Peut-on inventer une grammaire pour la réussite scolaire ? » in Brissaud. C \&Grossmann F. [coord], La construction des savoirs grammaticaux Paris, I.N.R.P., collection « Repères » $\mathrm{n}^{\circ} 39$ (17-41).

VOGÜÉ (DE), Sarah (1989) « La théorie des opérations énonciatives comme théorie de référence », Semen [en ligne] 4|1989, mis en ligne le 05 juin 2008, consulté le 25 mars 2015.URL : http:// semen.revues.org/6653 


\section{NOTES}

1. Le cycle 3 concerne les $\mathrm{CE} 2, \mathrm{CM} 1, \mathrm{CM} 2$ pendant ces dernières années où j'ai élaboré et testé les ateliers. Le cycle 3 regroupera CM1, CM2 et $6^{\text {ème }}$ à partir de la rentrée 2015.

2. Les A.P.C. sont des horaires librement affectés aux maitres, afin qu'ils regroupent, hors temps scolaire, par exemple le midi pendant une vingtaine de minutes, des élèves de leur choix. Ils composent des "groupes de besoin » : il ne s'agit donc pas uniquement de soutien aux élèves en difficulté : des élèves sans difficultés particulières peuvent être regroupés pour un autre besoin, par exemple celui d'approfondir une notion.

3. Il n'est évidemment pas question de proposer une "amélioration » du schéma de Jakobson, dont la communauté linguistique a montré les limites, mais de montrer sous forme de schéma comment fonctionne le langage dans le cadre d'une coénonciation. Les professeurs des écoles, non spécialistes, qui ont en tête le schéma critiqué, seront ainsi mieux à même de percevoir les réalités que je souhaite mettre en évidence.

4. Ce site n'est pas référencé sur les moteurs de recherche et fonctionne par identifiants : http:// ateliers-antoine.wix.com/ateliers-d-antoine

\section{RÉSUMÉS}

Après avoir montré la nécessité d'une reconfiguration didactique de l'enseignement de la grammaire en langue maternelle à l'école élémentaire française, cet article expliquera le dispositif que nous proposons en formation des enseignants du premier degré : les «Ateliers d'Antoine " sont conçus pour les élèves de CM1 et CM2 (8 à 10 ans) afin de les aider à développer une réflexion métalinguistique à partir de faits de langue problématisés, à l'aide d'un outil : le "carré-problème », et d'un rituel : le débat d'acceptabilité. La nouveauté de cette démarche repose sur le changement de cadre théorique : je fais l'hypothèse que la Théorie des Opérations Énonciatives d'Antoine Culioli est très adaptée aux besoins des enfants et, malgré la complexité de cette théorie, je propose de l'utiliser à l'école élémentaire, notamment pour une approche métalinguistique.

After showing the necessity to reconfigure the didactics of mother tongue grammar at the French elementary school level, this article describes the scheme I am suggesting to adopt in courses for teachers of elementary school. "Antoine's Workshops" have been devised for pupils in fourth and fifth grade -8 to 10 years of age - in order to help them develop a metalinguistic reflection about language problems. This is carried out with a special tool called the "problemsquare" and a special ritual : the "acceptability debate". The novelty of such an approach rests upon a change in the conceptual framework: I suggest that the "Theory of Enunciative Operations" by Antoine Culioli suits children's needs particularly well and despite its complexity I propose using it at elementary school level to provide teachers with reliable metalinguistic notions. 
INDEX

Keywords: Grammar, mother tongue, elementary school, French didactics, Antoine Culioli Mots-clés : Grammaire, langue maternelle, école élémentaire, didactique du français, Antoine Culioli

\section{AUTEUR}

\section{LIONEL AUDION}

E.S.P.É. - Université de Nantes, LLING - EA 382

lionel.audion@univ-nantes.fr 